\title{
Nonlinear Vibrations of an Uncertain Dual-Rotor Rolling Bearings System with Coupling Misalignment
}

\author{
Chao $\mathrm{Fu}^{1} \cdot{\mathrm{Kuan} \mathrm{Lu}^{1} \cdot \text { Yongfeng Yang }}^{1} \cdot$ Zhongliang Xie $^{1} \cdot$ Anbo Ming $^{1}$
}

Received: 26 December 2021 / Accepted: 16 February 2022 / Published online: 2 March 2022

(c) The Author(s) 2022

\begin{abstract}
In this paper, the nonlinear vibration responses of a dual-rotor system supported on the ball bearings considering coupling misalignment are investigated with inevitable uncertainties included. Moreover, uncertain parameters are modelled by non-probabilistic interval variables, alleviating the hash demands in fitting into a sophisticated probability law. It is then more suited for engineering problems that have sparse prior data on uncertainties. The deterministic vibration responses, orbits and frequency spectrum are provided first to exhibit the evolution of the vibrations. Then, several physical parameters are studied to reveal the effects of their uncertainty on the nonlinear vibrations at different rotating speeds. It is worth noting that uncertainty in the speed ratio between the higher-pressure and lower-pressure rotors has great impacts. Moreover, the sensitivity also depends on the rotating speed.
\end{abstract}

Keywords Dual-rotor - Interval uncertainty $\cdot$ Ball bearing $\cdot$ Coupling misalignment . Nonlinear response

\section{Abbreviations \\ FE Finite element \\ FEM Finite element method}

Chao Fu and Kuan Lu: Co-first authors.

Chao Fu

fuchao@nwpu.edu.cn

Kuan Lu

lukuan@nwpu.edu.cn

Yongfeng Yang

yyf@nwpu.edu.cn

Zhongliang Xie

zlxie@nwpu.edu.cn

Anbo Ming

anboming@nwpu.edu.cn

1 Institute of Vibration Engineering, Northwestern Polytechnical University, Xi' an 710072, China 


\section{Introduction}

Dual-rotor system structure is typically employed in aero-engines for its advantages over single rotor systems in several aspects. This type of dual-rotor system can be divided into a higher-pressure rotor and a lower-pressure rotor, connected by an inter-shaft bearing. Importantly, the rotors are supported and connected by bearings to stator structures, such as rolling bearings [1, 2] and journal bearings $[3,4]$. Due to the nature of contact reaction forces in such bearings, the dynamic characteristics exhibit nonlinearities [5-8]. Therefore, it is uneasy to fully understand the mechanism and theory of the vibration behaviours of such dual-rotor rolling bearings systems. Many researchers have done fruitful contributions on this topic aiming to provide better interpretation and guide the design and dynamic investigations [9-12]. Guskov et al. [13] carried out experimental and numerical studies of a dual-shaft test rig with inter-shaft bearing. The influences of each rotor's rotation on critical speeds and the corresponding vibration amplitudes were examined. Chen et al. [14] employed a surrogate model for the unbalance prediction of a dual-rotor system to save computational cost. In addition to the nonlinearity in rolling bearings, the dual-rotor systems often suffer from misalignment faults [15], which could be parallel misalignment or angular misalignment between shafts. This fault is common due to assembling errors or operation reasons, and it can cause unexpected vibrations and severe consequences [16]. Zhang et al. [17] investigated experimentally the dynamic responses of a coaxial dual-rotor system with supporting misalignment, where agreements were found between test results and those of a finite element (FE) model. Yang et al. [18] proposed a unified FE model for the full-flexible shaft-disk-drum system where the bolted joints is taking into consideration.

The formerly mentioned works all adopted deterministic physical models when investigating the dynamics of dual-rotor systems. However, it is gradually realized that for such complicated vibration systems there are plenty of uncertainty factors that induced vibration level increasing, unexplainable behaviours and inaccurate dynamic response estimations [19-22]. For instance, the manufacture errors, material degradations, external load disturbance and surface roughness [23-26]. Zhou et al. [27] pointed out that these variabilities in engineering can be treated as narrowly bounded interval variables. These uncertainties will cause unexpected deviations in the crucial performances of the rotor systems, such as reliability problems [28]. Guo et al. [29] carried out dynamics analyses of a L-shaped liquid-filled pipe considering interval uncertainty and experimental validations are also presented. Attempts to track the effects of the uncertainties in vibrational responses of rotating structures have been already made through different modelling techniques, including the stochastic analysis [30] and fuzzy approach [31]. Notably, Sinou and Jacquelin [32] studied the influences of the polynomial chaos expansion order when stochastically modelling an asymmetric rotor system, where coupling misalignment was considered. For certain dualrotor systems, it can be very difficult to gather enough prior statistics to build a well-fitted probabilistic model or find an appropriate set membership. Thus, the 
interval analysis methods, which requires much less information of the uncertain parameters, can be of interest in such occasions [33]. Wang et al. [34] used a Taylor interval expansion approach to quantify the propagation of non-random uncertainties in an aero-engine rotor system considering misalignment. Fu et al. [35] established a meta-model for the prediction of a dual-rotor system. The metamodel works in a non-intrusive form that allows alterations in models and factors to be considered but little modification to the already established uncertainty tracking and rotordynamics analysis codes. Further, Fu et al. [36] conducted a nonlinear analysis of the frequency responses of a dual-rotor rubbing system with an inter-shaft bearing. However, the nonlinearity in ball bearings and coupling misalignment are not included.

In the present study, we aim to use a non-intrusive method for the investigation of nonlinear vibrations of a dual-rotor ball bearings system. Importantly, the uncertainty in the ball bearings is considered, the effects of which are of nonlinear nature and still remain unclear at present. Firstly, the complex rotor model will be described, and the finite element method (FEM) is used to derive its motion equations. Secondly, the uncertainty analysis tool will be explained based on the interval representations. Thirdly, several key physical parameters are taken as uncertain variables and their influences on the nonlinear responses are revealed. Lastly, the summarization is given.

\section{Physical System}

In this paper, a dual-rotor rolling bearings system with coupling misalignment is used. The configuration of the system is given in Fig. 1. It typically consists of a lower-pressure and a higher-pressure rotor, both of which are hollow shafts and the lower-pressure rotor higher pressure rotor. The two rotors are connected by the intershaft bearing 4 and rotate with different speeds that are often incommensurable. Each rotor has two rigid discs, representing the compressor and turbine [37]. Ball bearings 1-3 support the rotors and connect the stators. The stiffness and damping

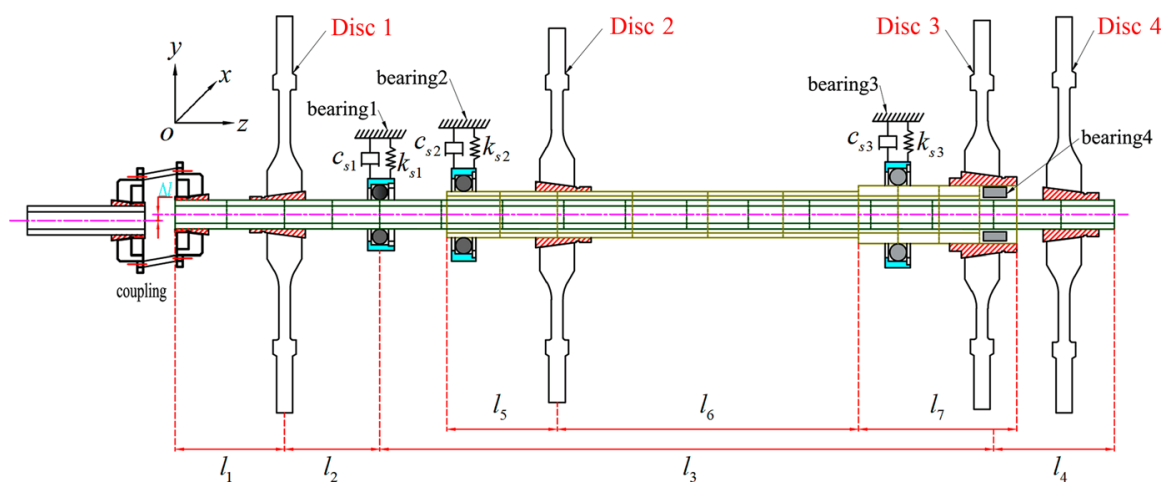

Fig. 1 Dual-rotor ball bearings system [37] 
Table 1 Disc parameters

\begin{tabular}{lllllll}
\hline No & $\begin{array}{l}\text { Inner diam- } \\
\text { eter }(\mathrm{mm})\end{array}$ & $\begin{array}{l}\text { Outer diam- } \\
\text { eter }(\mathrm{mm})\end{array}$ & Thickness $(\mathrm{mm})$ & Mass $(\mathrm{kg})$ & $\begin{array}{l}\text { Eccentric- } \\
\text { ity }(\mathrm{mm})\end{array}$ & $\begin{array}{l}\text { Moment of } \\
\text { inertia }(\mathrm{kg} \\
\left.\mathrm{m}^{2}\right)\end{array}$ \\
\hline Disc 1 & 12.5 & 62.5 & 20.0 & 10.52 & 0.030 & 0.072 \\
Disc 2 & 20.0 & 42.5 & 20.0 & 10.35 & 0.045 & 0.077 \\
Disc 3 & 20.0 & 42.5 & 20.0 & 10.35 & 0.030 & 0.066 \\
Disc 4 & 12.5 & 62.5 & 20.0 & 10.52 & 0.045 & 0.085 \\
\hline
\end{tabular}

Table 2 Bearing parameters

\begin{tabular}{lllllcl}
\hline No & $\begin{array}{l}\text { Inner ring } \\
\text { diameter } \\
(\mathrm{mm})\end{array}$ & $\begin{array}{l}\text { Outer ring } \\
\text { diameter } \\
(\mathrm{mm})\end{array}$ & $\begin{array}{l}\text { Ball } \\
\text { diameter } \\
(\mathrm{mm})\end{array}$ & $\begin{array}{l}\text { Number } \\
\text { of balls } \\
(-)\end{array}$ & Basic stiffness $\left(\mathrm{N} / \mathrm{m}^{3 / 2}\right)$ & $\begin{array}{l}\text { Radial } \\
\text { clearance } \\
(\mu \mathrm{m})\end{array}$ \\
\hline Bearing 1 & 23.38 & 15.43 & 7.935 & 8 & $3.85 \times 10^{9}$ & 30 \\
Bearing 2 & 30.87 & 22.85 & 8.01 & 11 & $4.36 \times 10^{9}$ & 5 \\
Bearing 3 & 36.66 & 29.63 & 7.018 & 18 & $5.47 \times 10^{9}$ & 6 \\
Bearing 4 & 23.31 & 17.29 & 6.012 & 16 & $11.42 \times 10^{9}$ & 8 \\
\hline
\end{tabular}

coefficients of the rolling bearings are illustrated in the model using $k_{s i}$ and $c_{s i}, i=1$, $2,3,4$. The lower-pressure rotor shaft is normally longer and has a smaller diameter than the shaft of the higher-pressure rotor, making it slender and more easily to vibrate heavily. The overall length of the lower-pressure rotor is $0.778 \mathrm{~m}$ with $l_{1}=0.094 \mathrm{~m}, l_{2}=0.09 \mathrm{~m}, l_{3}=0.5 \mathrm{~m}$ and $l_{4}=0.094 \mathrm{~m}$. Its inner diameter is $0.0075 \mathrm{~m}$ and its outer diameter is $0.0125 \mathrm{~m}$. The length of the higher-pressure rotor is $0.408 \mathrm{~m}$ with $l_{5}=0.08 \mathrm{~m}, l_{6}=0.208 \mathrm{~m}$ and $l_{7}=0.14 \mathrm{~m}$. Properties of the discs and ball bearings are listed in Tables 1 and 2. Moreover, the mass of the bearings 1-3 supports are $2.1 \mathrm{~kg}, 2.3 \mathrm{~kg}$ and $1.8 \mathrm{~kg}$, respectively. The mass of couplings for the two rotors is $0.75 \mathrm{~kg}$.

Parallel misalignment in the coupling is included in the model, which induces the reaction forces [38]:

$$
\left[\begin{array}{l}
F_{c x} \\
F_{c y}
\end{array}\right]=\left[\begin{array}{c}
2 m_{c} \Delta l \omega^{2} \cos (2 \omega t) \\
2 m_{c} \Delta l \omega^{2} \sin (2 \omega t)
\end{array}\right]
$$

where $m_{c}$ is the mass of the coupling, $t$ is time, $\omega$ represents the rotating speed and $\Delta l$ is the magnitude of parallel misalignment.

The whole system is modelled using the FEM with beam elements, bearing elements, rigid disc elements and coupling elements. Four degrees of freedom are considered for each node, i.e., two lateral displacements and two rotational angular displacements. The discs are treated as rigid bodies while their flexibility is neglected. Shear effects are included in the beam theory, which allows the consideration of torsional and lateral couplings. Detailed theory for establishing the motion equations of these elements can be found in classic textbooks and relevant publications [39-41]. The overall 
equation of motion of the system after assembling of elemental matrices is described by

$$
\mathbf{M} \ddot{\mathbf{q}}+(\mathbf{C}-\omega \mathbf{G}) \dot{\mathbf{q}}+\mathbf{K q}+\mathbf{F}_{b}(t, \mathbf{q})=\mathbf{F}_{g}+\mathbf{F}_{u}(t)+\mathbf{F}_{m}(t)
$$

where notations $\mathbf{M}, \mathbf{K}, \mathbf{C}, \mathbf{G}$ represent the global mass, stiffness, damping and gyroscopic matrices of the complete dual-rotor bearings system. $\mathbf{q}$ is the displacement vector and dot over it means the derivation operation over time. Proportional damping is used besides damping provided by the ball bearings. Excitations on the system are the gravitational forces $\mathbf{F}_{g}$, unbalance forces $\mathbf{F}_{u}$ and misalignment forces $\mathbf{F}_{m}$. The nonlinear reaction forces of ball bearings are denoted by $\mathbf{F}_{b}(t, \mathbf{q})$ and are expressed as [42]

$$
\left[\begin{array}{c}
F_{x_{i}} \\
F_{y_{i}}
\end{array}\right]=C_{b_{i}} \sum_{j=1}^{N_{b_{i}}} H\left(\delta_{i j}\right) \delta_{i j}^{n}\left[\begin{array}{c}
\cos \theta_{i j} \\
\sin \theta_{i j}
\end{array}\right], \theta_{i j}=\frac{2 \pi(j-1)}{N_{b_{i}}}+\Omega_{i} t, \delta_{i j}=x_{i} \cos \theta_{i j}+y_{i} \sin \theta_{i j}-\delta_{i 0}
$$

where $\theta_{i j}, \delta_{i j}$ are the instantaneous angle and contact deformation of the $i$ th and $j$ th rolling bodies, $n=3 / 2$ is the Hertz contact nonlinearity, $H\left(\delta_{i j}\right)$ is the Heaviside function, $C_{b_{i}}, N_{b_{i}}, \Omega_{i}, 2 \delta_{i 0}$ describe the contact stiffness, number of roller bodies, cage speed, initial radial clearance of the $i$ th bearing.

The dynamical system given in Eq. (3) is nonlinear by nature caused by the contact forces in ball bearings. Obtaining accurate solutions to it is quite challenging as the dual-rotor system is rather complicated. When the uncertainty is included, the problem becomes even more difficult. However, the smooth and precise estimations of nonlinear responses of the system will certificate the merits of the uncertainty tracking method in dealing with complex nonlinear vibration systems.

\section{Uncertainty Propagation}

The interval uncertainties in such a complex dual-rotor system shown in the previous section can be introduced in different sources and may be difficult to track. Subsequently, the quantification of these uncertainties in the nonlinear dynamic response to a satisfactory level cannot be achieved by most of the existing methods. The Chebyshev inclusion function proposed by $\mathrm{Wu}$ et al. [43] can be applied to study the nonlinear responses of a mechanical system from a black-box point of view. It soon witnessed many applications in different uncertain nonlinear vibration systems. Based on this theory, the nonlinear interval response (any nodal displacement) is approximated by the Chebyshev orthogonal series. For example, let us predefine a set of interval uncertain quantities in the dual-rotor bearings system $\phi$ as

$$
\boldsymbol{\phi}=\left[\phi_{1}, \phi_{2}, \ldots, \phi_{n}\right]^{\mathrm{T}}
$$

where $n$ is the number of interval variables. The element in $\phi$ can be expressed as

$$
\phi_{i}^{I}=\left[\phi_{i}^{c}-\beta \phi_{i}^{c}, \phi_{i}^{c}+\beta \phi_{i}^{c}\right], i=1,2, \ldots, n
$$


where $\phi_{i}^{c}$ is the middle value of the interval quantity and $\beta$ is its variation coefficient. Thus, the motion equation of the dual-rotor bearings system should be given in the following form to include the effects of uncertain variables

$$
\mathfrak{J}:\left\{\begin{array}{l}
\mathbf{M} \ddot{\mathbf{q}}+(\mathbf{C}-\omega \mathbf{G}) \dot{\mathbf{q}}+\mathbf{K q}+\mathbf{F}_{b}(t, \mathbf{q})=\mathbf{F}_{g}+\mathbf{F}_{u}(t)+\mathbf{F}_{m}(t) \\
\mathbf{M}, \mathbf{C}, \mathbf{G}, \mathbf{K}, \mathbf{q}, \mathbf{F}_{g}, \mathbf{F}_{u}, \mathbf{F}_{m} \text { depends on } t, \mathbf{q}, \boldsymbol{\phi}
\end{array}\right\}
$$

The above formulation of the uncertain system $\mathfrak{I}$ indicates that the system matrices including excitations will depend on the variations of uncertainties based on what interval parameters are considered and they are not fully specified yet, which prevents it from being solved by traditional numerical methods directly. Based on the multi-element Chebyshev approximation theory, the unknown relationship $\mathfrak{I}$ between the uncertain variables and the nonlinear responses can be expressed by [43]

$$
\mathfrak{\Im}(\boldsymbol{\phi}, t)=\sum_{k_{1}=0}^{r} \cdots \sum_{k_{n}=0}^{r} \frac{1}{2^{p}} \xi_{k_{1}, \cdots, k_{n}} C_{k_{1}, \cdots, k_{n}}(\boldsymbol{\theta})
$$

where $r$ denotes the expansion order, $\xi_{k_{1}, \cdots, k_{n}}$ is the expansion coefficient to be determined, $p$ represents the appearance times of 0 in the index set $k_{1}, \cdots, k_{n}, C_{k_{1}, \cdots, k_{n}}$ is the $n$-dimensional Chebyshev series as

$$
C_{k_{1}, \cdots, k_{n}}(\boldsymbol{\theta})=\cos \left(k_{1} \theta_{1}\right) \cos \left(k_{2} \theta_{2}\right) \cdots \cos \left(k_{n} \theta_{n}\right)
$$

The connection between the physical uncertain parameter set $\phi$ and standard interval variable set $\boldsymbol{\theta}$ with all elements defined in $[-1,1]$ is

$$
\theta=\arccos \frac{2 \underline{\phi}-(\bar{\phi}+\underline{\phi})}{\bar{\phi}-\underline{\phi}}, \theta \in[0, \pi]^{n}, \phi \in[\underline{\phi}, \bar{\phi}]^{n}
$$

where $\phi, \bar{\phi}$ are the lower and upper bound vector of $\phi$.

Now, the unknown coefficients need to be solved to completely establish the approximation model. The Gauss-Chebyshev formulas can be applied for this purpose

$$
\xi_{k_{1}, \cdots, k_{n}}=\sum_{j_{1}=0}^{\lambda} \cdots \sum_{j_{n}=0}^{\lambda}\left(\frac{2}{\lambda}\right)^{n} \tilde{\mathbf{q}}\left(t, \cos \tilde{\theta}_{j_{1}}, \cdots, \cos \tilde{\theta}_{j_{n}}\right) \cos \left(k_{1} \tilde{\theta}_{j_{1}}\right) \cdots \cos \left(k_{r} \tilde{\theta}_{j_{n}}\right)
$$

where $\tilde{\theta}_{j}$ is the interpolation point set and $\lambda$ is the number of interpolations for each dimension. From the function approximation principle, the number of interpolations should be larger than the order, i.e., the following should be met

$$
\lambda \geq r+1
$$

The sampled nonlinear response $\tilde{\mathbf{q}}\left(t, \cos \tilde{\theta}_{j_{1}}, \cdots, \cos \tilde{\theta}_{j_{n}}\right)$ is extracted from the previously constructed deterministic dual-rotor bearings model using the fourth-order 
Runge-Kutta method. The notation $\cos \left(k_{1} \tilde{\theta}_{j_{1}}\right) \cdots \cos \left(k_{r} \tilde{\theta}_{j_{n}}\right)$ is the value of the Chebyshev series at interpolations. Specifically, we used the Chebyshev zeros as the interpolation points, which can be given by

$$
\theta_{j}=\frac{2 j-1}{2 \lambda} \pi, j=1,2, \ldots, \lambda
$$

Since now the approximation relationship is justified completely, the interval response can be given by finding the bounds of the surrogate function:

$$
\begin{gathered}
\underline{\mathbf{q}}=\frac{1}{2^{n}} \xi_{0, \cdots, 0}-\sum_{\substack{0 \leq k_{1}, \cdots, k_{n} \leq n \\
k_{1}+\cdots+k_{n} \geq 1}} \frac{1}{2^{p}}\left|\xi_{k_{1}, \cdots, k_{n}}\right| \\
\overline{\mathbf{q}}=\frac{1}{2^{n}} \xi_{0, \cdots, 0}+\sum_{\substack{0 \leq k_{1}, \cdots, k_{n} \leq n \\
k_{1}+\cdots+k_{n} \geq 1}} \frac{1}{2^{p}}\left|\xi_{k_{1}, \cdots, k_{n}}\right| \\
\mathbf{q}^{I}=[\underline{\mathbf{q}}, \overline{\mathbf{q}}]
\end{gathered}
$$

where $p$ has the same meaning as before, a superscript $I$ denotes the interval output and $\mathbf{q}, \overline{\mathbf{q}}$ represent the two bounds of the nonlinear responses.

\section{Numerical Results}

In this section, numerical investigations are carried out based on the previously explained theory. To provide the deterministic results, i.e., the behaviours of the dual-rotor system without uncertainty, all the parameters are kept constant, and the motion equations are solved using the Runge-Kutta integration. Here, we present the vibration responses of the nodes at bearing \#1 and bearing \#3 when the rotating speed of the lower-pressure rotor is $90 \pi \mathrm{rad} / \mathrm{s}$, as shown in Figs. 2 and 3. It is seen from Fig. 2a that the time history of bearing \#1 in the $X$-direction shows a little distortion of the waveform. However, it is close to the harmonic vibrations, as also evidenced by the orbit in Fig. 2b. The frequency spectrum given in Fig. 2c reveals the root mechanism, i.e., the frequency component with the most significant amplitude is the synchronous vibrations. Other components induced by the nonlinear ball bearings and the misalignment are very weak. On the contrary, the results for the same rotation speed of bearing \#3 exhibit more complexity, as plotted in Fig. 3. The responses in Fig. 3a is nonlinear by nature and the orbit shown in Fig. $3 b$ is complicated but still clear. Looking at the frequency spectrum in Fig. 3c, we know that the main components are the rotating frequency as well as its fractal and $2 \mathrm{X}$ frequency. This is typically caused by ball bearings and the misalignment fault. When the rotation speed is $100 \pi \mathrm{rad} / \mathrm{s}$, the respective vibration characteristics of bearing \#1 and bearing \#3 are illustrated in Figs. 4 and 5. From the two diagrams, we can notice that for a higher rotating speed compared with previous results, the 

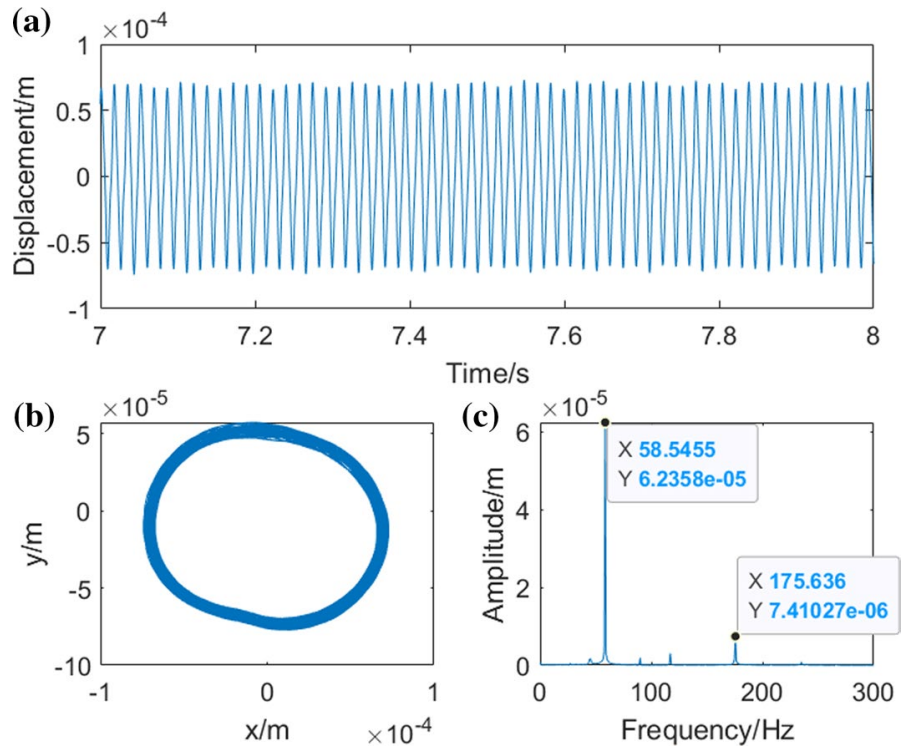

Fig. 2 Vibration responses of the dual-rotor system at bearing $1(\omega=90 \pi \mathrm{rad} / \mathrm{s})$ : a time history in the $X$ direction, $\mathbf{b}$ orbit and $\mathbf{c}$ frequency spectrum
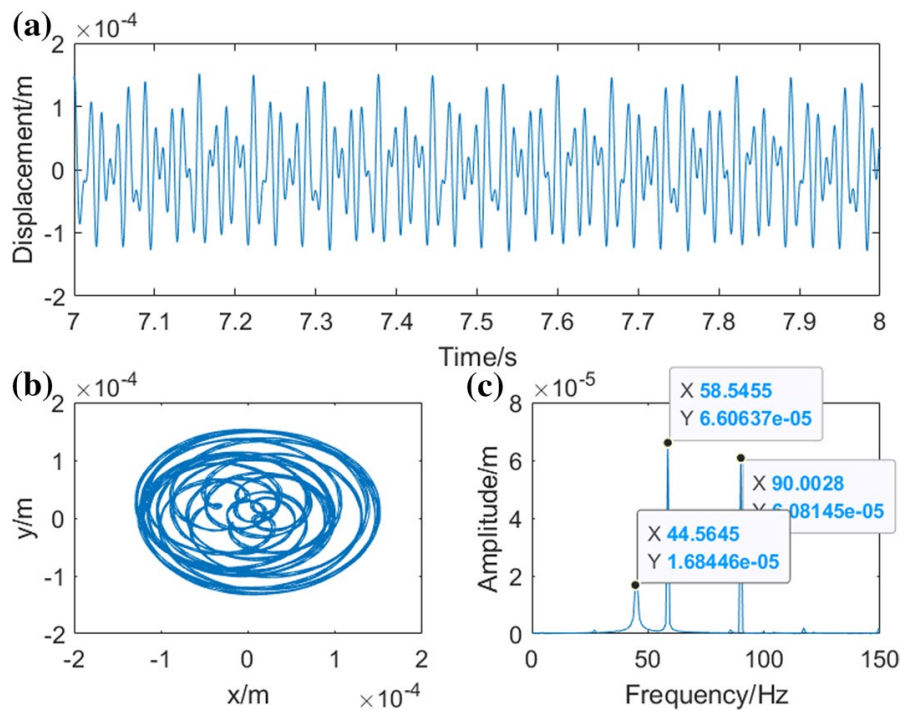

Fig. 3 Vibration responses of the dual-rotor system at bearing $3(\omega=90 \pi \mathrm{rad} / \mathrm{s})$ : a time history in the X direction, $\mathbf{b}$ orbit and $\mathbf{c}$ frequency spectrum

vibrational behaviours of the two bearings are much more complex. The time history shows strong nonlinearities, and the orbit has no clear path. The Fourier transform of the displacements gives multiple significant components corresponding to 

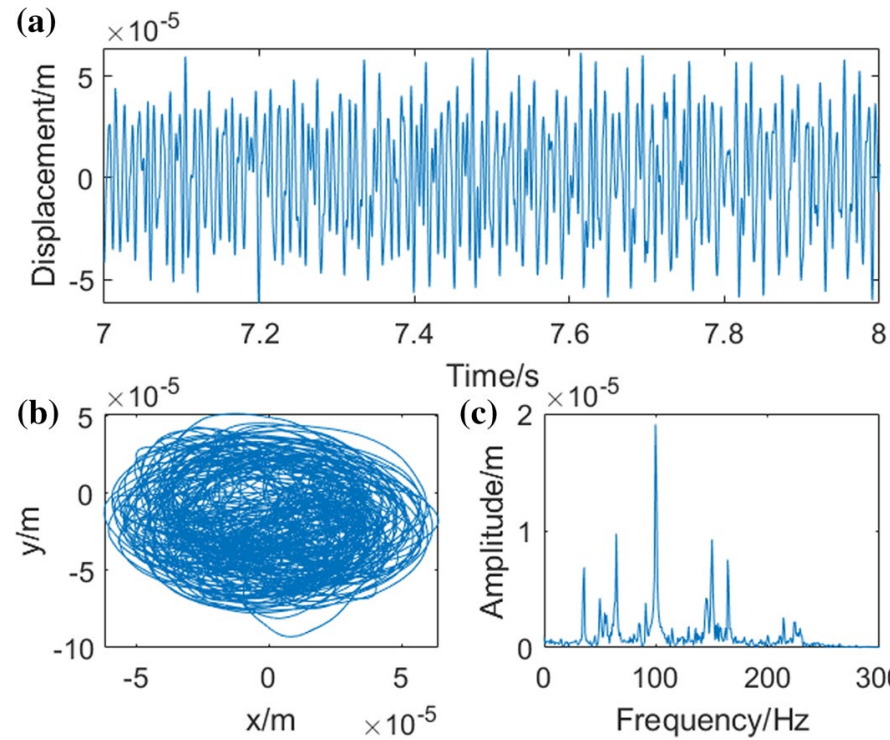

Time/s

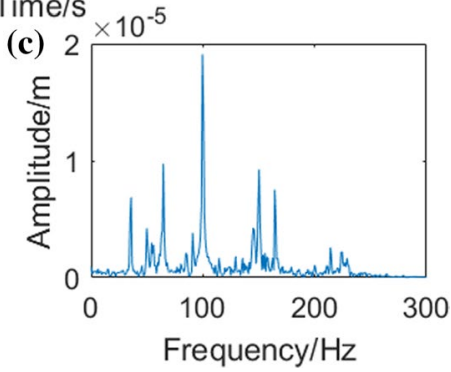

Fig. 4 Vibration responses of the dual-rotor system at bearing $1(\omega=100 \pi \mathrm{rad} / \mathrm{s})$ : a time history in the $\mathrm{X}$ direction, $\mathbf{b}$ orbit and $\mathbf{c}$ frequency spectrum
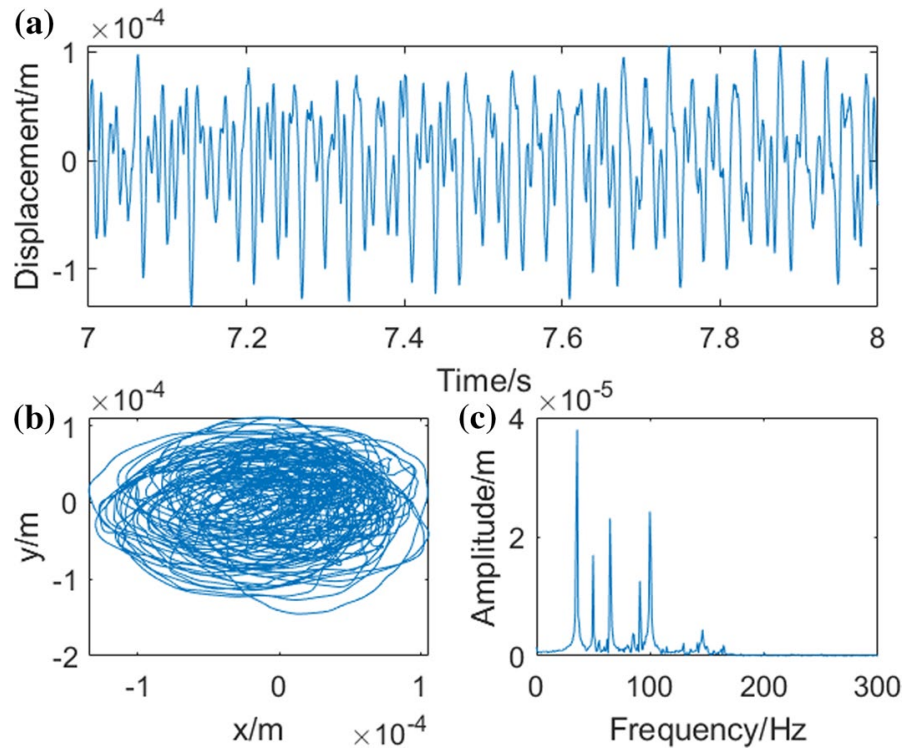

Fig. 5 Vibration responses of the dual-rotor system at bearing $3(\omega=100 \pi \mathrm{rad} / \mathrm{s})$ : a time history in the X direction, $\mathbf{b}$ orbit and $\mathbf{c}$ frequency spectrum 
the unbalance excitation, ball bearings nonlinearity and the parallel misalignment fault. It is therefore very meaningful to quantify the propagating of various uncertainties in such nonlinear vibrations and this study is of course a great challenge for the uncertainty method used. The results with different interval parameters considered will be presented. It should be noted that the interval variability levels used in this paper are typical values frequently adopted in literature. However, one can choose to analysis any reasonable variability levels based on their own needs as the procedure proposed in the current study is non-intrusive, i.e., it can be realized by only varying the uncertainty coefficients.

The speed ratio between the lower-pressure and higher-pressure rotors is very important for engineers in designing an actual aero-engine dual-rotor system operating at $90 \pi \mathrm{rad} / \mathrm{s}$. Thus, the variability in this parameter could be of great importance. For this reason, $10 \%$ uncertainty is considered in the speed ratio and the vibration response is demonstrated in Fig. 6. It appears that the speed ratio uncertainty has great impacts on the nonlinear vibrations. Although the deterministic responses show trivial nonlinear nature, the bounds of the responses exhibit much complexity and multiple peaks. The response interval enclosed is wide, indicating the high impacts of the uncertainty. It is caused by the high sensitivity of the system to the fluctuations of the speed ratio and is also sensitive to the change of the rotating speed. Quantitatively, the deterministic vibration peak has evolved to an interval of $[67.01,87.79] \mu \mathrm{m}$, an uplifting of $31 \%$ from the lower bound to the upper bound. Further, the uncertainties with the same interval coefficient of $10 \%$ in the

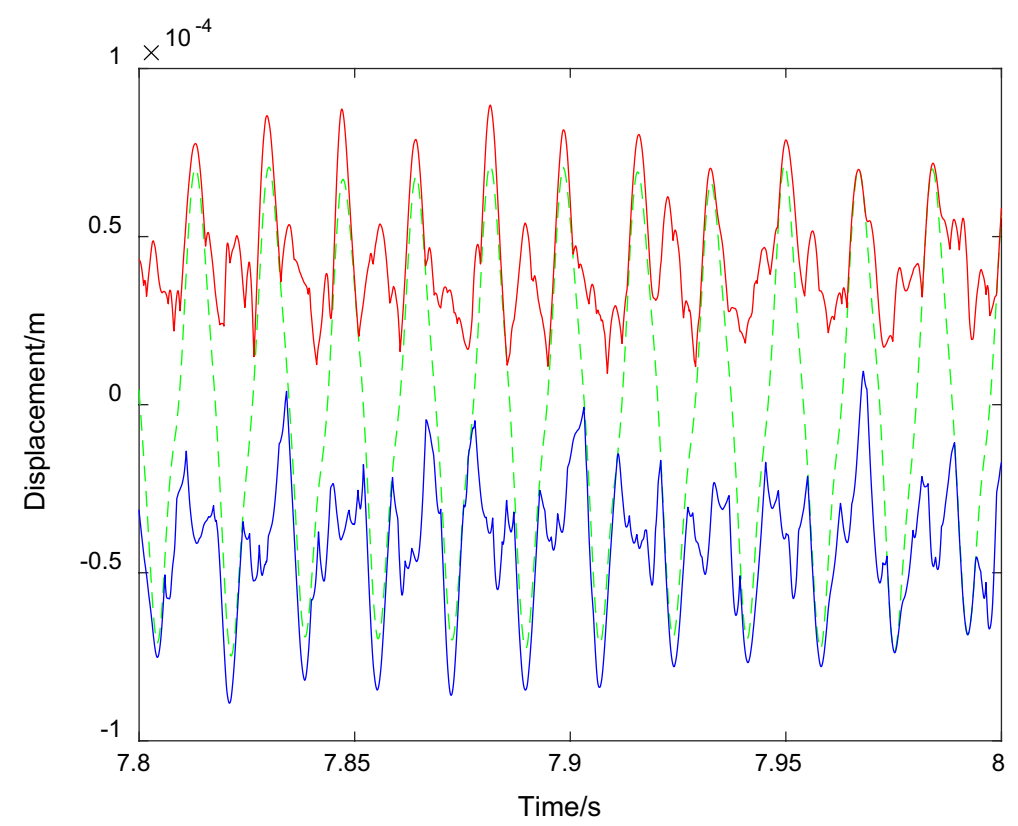

Fig. 6 Vibration responses of the dual-rotor system with $10 \%$ uncertainty in speed ratio $(\omega=90 \pi \mathrm{rad} / \mathrm{s})$ : dashed green line-deterministic curve, blue solid line-lower bound and red solid line-upper bound 

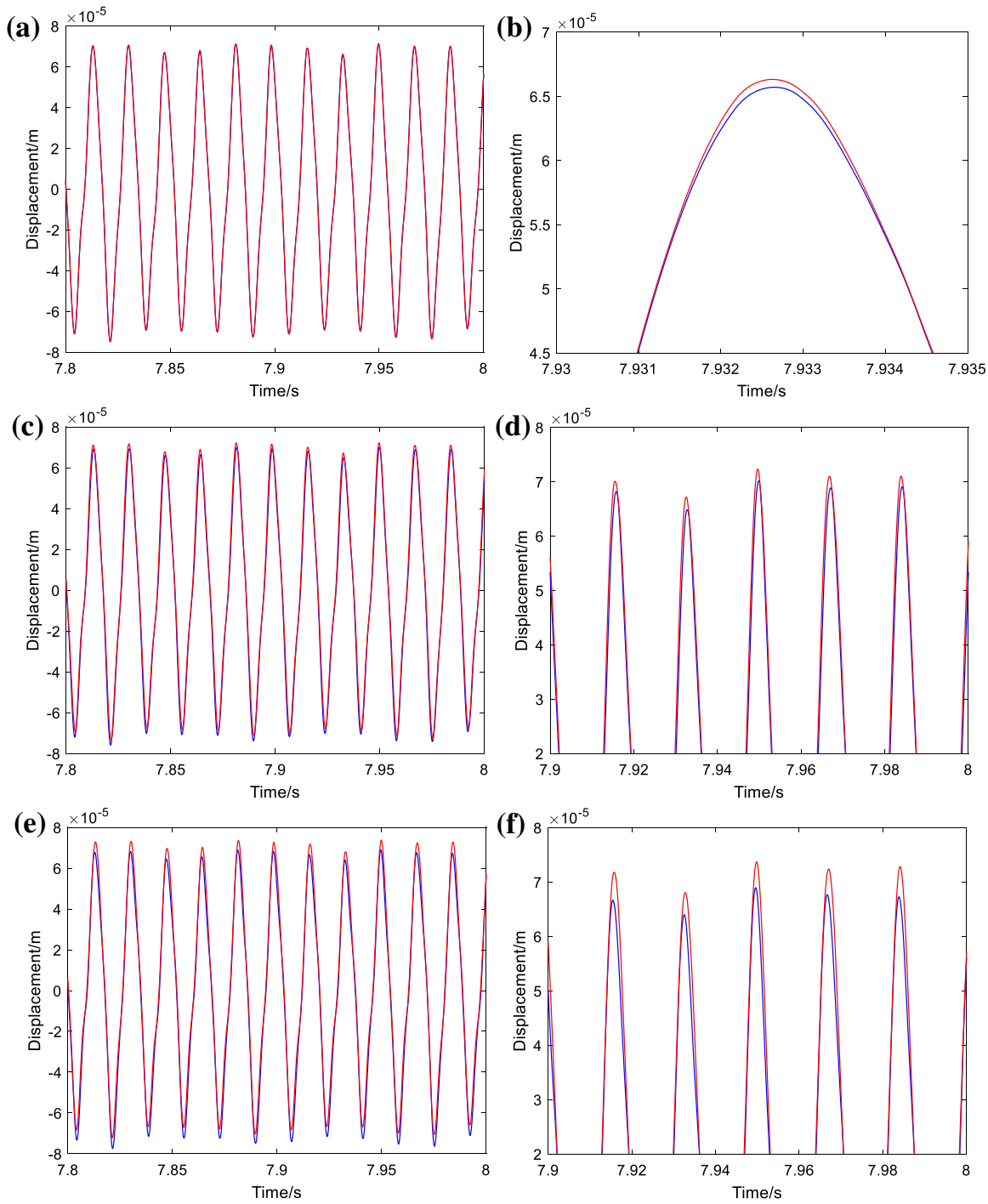

Fig. 7 Vibration responses of the dual-rotor system with different uncertainties $(\omega=90 \pi \mathrm{rad} / \mathrm{s})$ : blue solid line-lower bound and red solid line-upper bound; a $10 \%$ uncertainty in misalignment amplitude, b zoomed view of a, c $10 \%$ uncertainty in unbalance of higher-pressure rotor, $\mathbf{d}$ zoomed view of $\mathbf{c}, \mathbf{e} 10 \%$ uncertainty in the clearance of bearing \#1 and $\mathbf{f}$ zoomed view of $\mathbf{e}$

misalignment magnitude, unbalance of the higher-pressure rotor and the radial clearance of bearing \#1 are investigated and the interval responses are given in Fig. 7 with zoomed local views. We can find from the response intervals that the uncertainties in these parameters caused relatively small impacts compared with the uncertain parameter ratio. In addition, the nonlinearity in the dynamic responses is not presented by large. The severity of response fluctuations due to these uncertainties 
increase from the misalignment, unbalance to bearing clearance. Importantly, the only obvious deviations are observed near peaks. While the other deviations seem linear, the response positions of peaks are slightly shifted.

Further, the dynamic response intervals of bearing \#1 when the rotating speed is $100 \pi \mathrm{rad} / \mathrm{s}$ considering $2 \%$ uncertainties in the four investigated cases. The corresponding interval responses are plotted in Fig. 8. As expected, the vibration responses show complicated characteristics due to uncertainty. The upper and lower bounds have many peaks and local fluctuations, which are not necessarily present in the deterministic response curves but induced by variabilities of parameters. The total increments from the lower peaks to the upper ones are nearly tripled, indicating the severe propagation of uncertainty and very high sensitivity of the system responses to the corresponding uncertainty. To be noted, in this case, the uncertainty coefficient is small. It is, therefore, demonstrated that small deviations in parameters can lead to heavy fluctuations in the dynamic response in nonlinear and faulted dualrotor systems. What's more, the peaks and local fluctuations of the different cases happen at different time steps, revealing their different propagating mechanism in the nonlinear vibrations. It is reasonable to estimate that the combined deviations
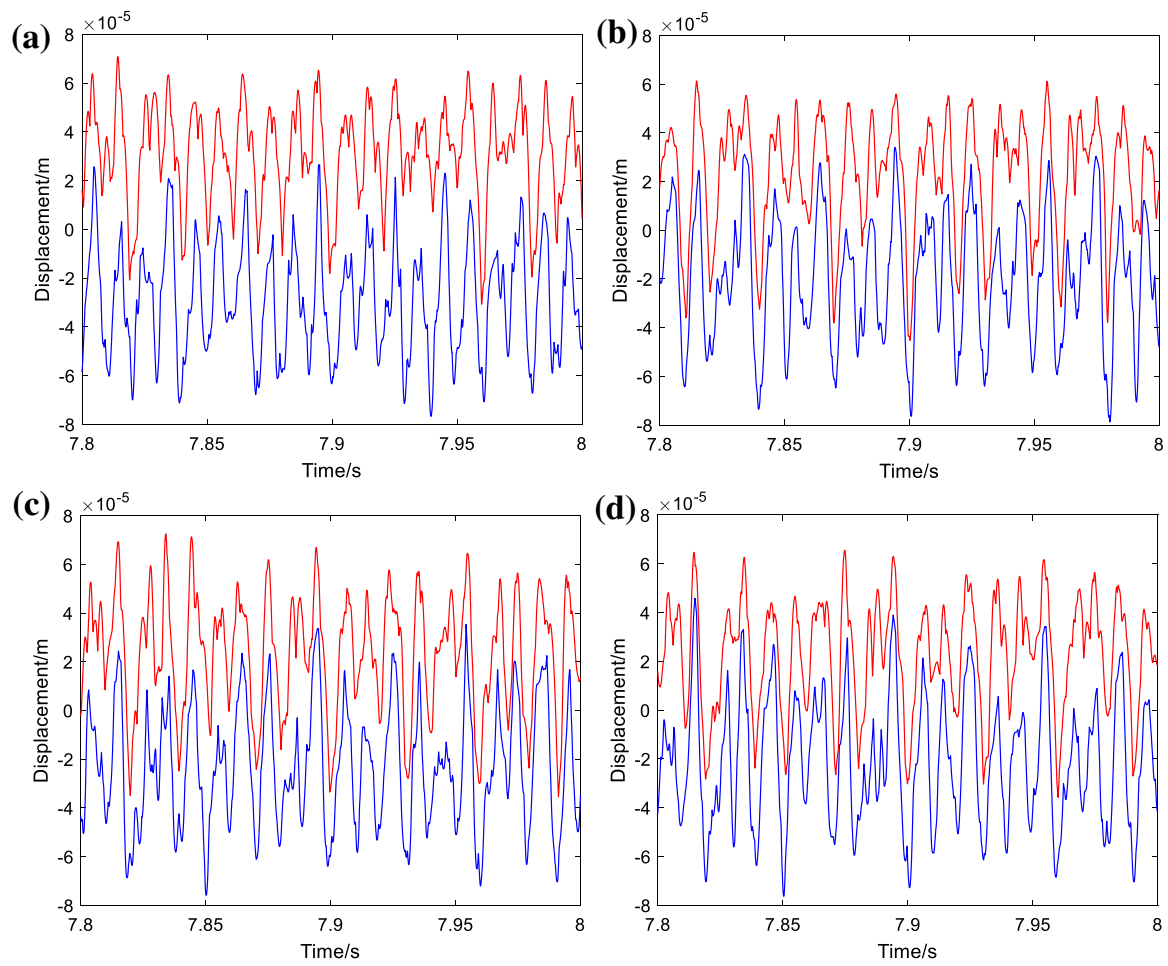

Fig. 8 Vibration responses of the dual-rotor system with different uncertainties $(\omega=100 \pi \mathrm{rad} / \mathrm{s})$ : Blue solid line-lower bound and red solid line-upper bound; a $2 \%$ uncertainty in misalignment amplitude, b $2 \%$ uncertainty in speed ratio, c $2 \%$ uncertainty in unbalance of higher pressure rotor and d $2 \%$ uncertainty in the clearance of bearing \#1 
can be even more striking. Thus, it is important to include irreducible uncertainty in robust design and trustworthy dynamic analysis of such dual-rotor ball bearing systems.

\section{Conclusion}

A dual-rotor ball bearings system with coupling misalignment and interval uncertainty is studied to reveal its nonlinear vibration characteristics. The presented result show important impacts of the interval physical parameters, especially the influence of the uncertain speed ratio. It is found that the nonlinear vibrations of the dual-rotor system are more complex at $100 \pi \mathrm{rad} / \mathrm{s}$ than $90 \pi \mathrm{rad} / \mathrm{s}$ with the vibrations at bearing \#3 more affected by the ball bearings and misalignment than at bearing \#1. Consequently, the uncertain response bounds are very complicated at $100 \pi \mathrm{rad} / \mathrm{s}$ even for a small uncertainty in the model. Local fluctuations and peaks are observed, which is induced by the nonlinear nature and effects of the uncertainties. This is related to the inherent vibration behaviors of the system at different rotating speeds. The findings in this study could provide references in analysing these types of systems and the reliable design of engineering dual-rotor systems. In future, coupling effects of the parametric uncertainty and nonlinearity in the dual-rotor system induced by the ball bearings or relevant faults such as cracks and rubbing need to be investigated, which will bring more thorough understandings.

Author Contributions CF: Conception, Methodology, Investigation and Writing. KL: Conception, Coding and Funding Acquisition. YY: Supervision, Validation and Reviewing. ZX: Validation and Reviewing. AM: Investigation and Reviewing.

Funding This work was sponsored by the Shanghai Sailing Program (Grant no. 22YF1452000), the Fundamental Research Funds for the Central Universities (Grant no. G2021KY0601) and the National Natural Science Foundation of China (Grant nos. 11972295 and 12072263).

Data Availability Statement Not applicable.

\section{Declarations}

Conflict of interest The authors declare that they have no conflict of interest.

Open Access This article is licensed under a Creative Commons Attribution 4.0 International License, which permits use, sharing, adaptation, distribution and reproduction in any medium or format, as long as you give appropriate credit to the original author(s) and the source, provide a link to the Creative Commons licence, and indicate if changes were made. The images or other third party material in this article are included in the article's Creative Commons licence, unless indicated otherwise in a credit line to the material. If material is not included in the article's Creative Commons licence and your intended use is not permitted by statutory regulation or exceeds the permitted use, you will need to obtain permission directly from the copyright holder. To view a copy of this licence, visit http://creativecommons.org/licen ses/by/4.0/. 


\section{References}

1. Lu, Z., Zhong, S., Chen, H., Wang, X., Han, J., Wang, C.: Nonlinear response analysis for a dualrotor system supported by ball bearing. Int. J. Non-Linear Mech. 128, 103627 (2021)

2. He, D., Yang, Y., Xu, H., Ma, H., Zhao, X.: Dynamic analysis of rolling bearings with roller spalling defects based on explicit finite element method and experiment. J. Nonlinear Math. Phys. (2022). https://doi.org/10.1007/s44198-022-00027-y

3. Ma, J., Zhang, H., Lou, S., Chu, F., Shi, Z., Gu, F., Ball, A.D.: Analytical and experimental investigation of vibration characteristics induced by tribofilm-asperity interactions in hydrodynamic journal bearings. Mech. Syst. Signal Process. 150, 107227 (2021)

4. Xie, Z., Zhu, W.: Theoretical and experimental exploration on the micro asperity contact load ratios and lubrication regimes transition for water-lubricated stern tube bearing. Tribol. Int. 164, 107105 (2021)

5. Gupta, K., Gupta, K.D., Athre, K.: Unbalance response of a dual rotor system: theory and experiment. J. Vib. Acoust. 115(4), 427-435 (1993)

6. Zhang, Z., Rui, X., Yang, R., Chen, Y.: Control of period-doubling and chaos in varying compliance resonances for a ball bearing. J. Appl. Mech. 87(2), 021005 (2019)

7. Zhang, H., Lu, K., Zhang, W., Fu, C.: Investigation on dynamic behaviors of rotor system with looseness and nonlinear supporting. Mech. Syst. Signal Process. 166, 108400 (2022)

8. Xie, Z., Wang, X., Zhu, W.: Theoretical and experimental exploration into the fluid structure coupling dynamic behaviors towards water-lubricated bearing with axial asymmetric grooves. Mech. Syst. Signal Process. 168, 108624 (2022)

9. Ma, X., Ma, H., Qin, H., Guo, X., Zhao, C., Yu, M.: Nonlinear vibration response characteristics of a dual-rotor-bearing system with squeeze film damper. Chin. J. Aeronaut. 34(10), 128-147 (2021)

10. Tehrani, G.G., Gastaldi, C., Berruti, T.M.: Stability analysis of a parametrically excited ball bearing system. Int. J. Non-Linear Mech. 120, 103350 (2020)

11. Tiwari, M., Gupta, K., Prakash, O.: Effect of radial internal clearance of a ball bearing on the dynamics of a balanced horizontal rotor. J. Sound Vib. 238(5), 723-756 (2000)

12. Wang, N., Jiang, D., Xu, H.: Dynamic characteristics analysis of a dual-rotor system with inter-shaft bearing. Proc. Inst. Mech. Eng. Part G J. Aerosp. Eng. 233(3), 1147-1158 (2019)

13. Guskov, M., Sinou, J.-J., Thouverez, F., Naraikin, O.: Experimental and numerical investigations of a dual-shaft test rig with intershaft bearing. Int. J. Rotat. Mach. 2007, 075762 (2007)

14. Chen, X., Zhang, H., Zou, C., Zhai, J., Han, Q.: Research on the prediction method of unbalance responses of dual-rotor system based on surrogate models. SN Appl. Sci. 2(1), 1-13 (2020)

15. Kumar, A., Kumar, R.: Development of LDA based indicator for the detection of unbalance and misalignment at different shaft speeds. Exp. Tech. 44(2), 217-229 (2020)

16. Desouki, M., Sassi, S., Renno, J., Gowid, S.A.: Dynamic response of a rotating assembly under the coupled effects of misalignment and imbalance. Shock Vib. 2020, 8819676 (2020)

17. Zhang, H., Li, X., Yang, D., Jiang, L.: Vibration responses of a coaxial dual-rotor system with supporting misalignment. Appl. Sci. 11(23), 11219 (2021)

18. Yang, T., Ma, H., Qin, Z., Guan, H., Xiong, Q.: Coupling vibration characteristics of the shaft-diskdrum rotor system with bolted joints. Mech. Syst. Signal Process. 169, 108747 (2022)

19. Fu, C., Xu, Y., Yang, Y., Lu, K., Gu, F., Ball, A.: Response analysis of an accelerating unbalanced rotating system with both random and interval variables. J. Sound Vib. 466, 115047 (2020)

20. Zhou, B., Zi, B., Li, Y., Zhu, W.: Hybrid compound function/subinterval perturbation method for kinematic analysis of a dual-crane system with large bounded uncertainty. J. Comput. Nonlinear Dynam. 16(1), 014501 (2021)

21. Fu, C., Xu, Y., Yang, Y., Lu, K., Gu, F., Ball, A.: Dynamics analysis of a hollow-shaft rotor system with an open crack under model uncertainties. Commun. Nonlinear Sci. Num. Simul. 83, 105102 (2020)

22. Fu, C., Zhu, W., Yang, Y., Zhao, S., Lu, K.: Surrogate modeling for dynamic analysis of an uncertain notched rotor system and roles of Chebyshev parameters. J. Sound Vib. 524, 116755 (2022)

23. Ma, J., Fu, C., Zhu, W., Lu, K., Yang, Y.: Stochastic analysis of lubrication in misaligned journal bearings. J. Tribol. 144(8), 081802 (2022)

24. Liu, J., Pang, R., Xu, Y., Ding, S., He, Q.: Vibration analysis of a single row angular contact ball bearing with the coupling errors including the surface roundness and waviness. Sci. China Technol. Sci. 63(6), 943-952 (2020) 
25. Ma, J., Fu, C., Zhang, H., Chu, F., Shi, Z., Gu, F., Ball, A.D.: Modelling non-Gaussian surfaces and misalignment for condition monitoring of journal bearings. Measurement 174, 108983 (2021)

26. Gao, J., Zhou, B., Zi, B., Qian, S., Zhao, P.: Kinematic uncertainty analysis of a cable-driven parallel robot based on an error transfer model. J. Mech. Robot. 14(5), 051008 (2022)

27. Zhou, B., Zi, B., Qian, S.: Dynamics-based nonsingular interval model and luffing angular response field analysis of the DACS with narrowly bounded uncertainty. Nonlinear Dyn. 90(4), 2599-2626 (2017)

28. Zhang, Y., Liu, Y.: Modeling of the rotor-bearing system and dynamic reliability analysis of rotor's positioning precision. Proc. Inst. Mech. Eng. Part O J. Risk Reliabil. 235(3), 491-508 (2021)

29. Guo, X., Cao, Y., Ma, H., Xiao, C., Wen, B.: Dynamic analysis of an L-shaped liquid-filled pipe with interval uncertainty. Int. J. Mech. Sci. 217, 107040 (2022)

30. Li, Z., Jiang, J., Tian, Z.: Stochastic dynamics of a nonlinear misaligned rotor system subject to random fluid-induced forces. J. Comput. Nonlinear Dynam. 12(1), 011004 (2017)

31. Murugan, S., Ganguli, R., Harursampath, D.: Aeroelastic response of composite helicopter rotor with random material properties. J. Aircr. 45(1), 306-322 (2008)

32. Sinou, J.-J., Jacquelin, E.: Influence of Polynomial Chaos expansion order on an uncertain asymmetric rotor system response. Mech. Syst. Signal Process. 50, 718-731 (2015)

33. Rao, S.S., Berke, L.: Analysis of uncertain structural systems using interval analysis. AIAA J. 35(4), 727-735 (1997)

34. Wang, C., Ma, Y., Zhang, D., Hong, J.: Interval analysis on aero-engine rotor system with misalignment. In: American Society of Mechanical Engineers, V07AT30A002 (2015)

35. Fu, C., Feng, G., Ma, J., Lu, K., Yang, Y., Gu, F.: Predicting the dynamic response of dual-rotor system subject to interval parametric uncertainties based on the non-intrusive metamodel. Mathematics 8(5), 736 (2020)

36. Fu, C., Zhu, W., Zheng, Z., Sun, C., Yang, Y., Lu, K.: Nonlinear responses of a dual-rotor system with rub-impact fault subject to interval uncertain parameters. Mech. Syst. Signal Process. 170, 108827 (2022)

37. Lu, K., Jin, Y., Huang, P., Zhang, F., Zhang, H., Fu, C., Chen, Y.: The applications of POD method in dual rotor-bearing systems with coupling misalignment. Mech. Syst. Signal Process. 150, 107236 (2021)

38. Xu, M., Marangoni, R.: Vibration analysis of a motor-flexible coupling-rotor system subject to misalignment and unbalance, Part I: theoretical model and analysis. J. Sound Vib. 176(5), 663-679 (1994)

39. Li, Y., Cao, H., Tang, K.: A general dynamic model coupled with EFEM and DBM of rolling bearing-rotor system. Mech. Syst. Signal Process. 134, 106322 (2019)

40. Li, L., Luo, Z., He, F., Sun, K., Yan, X.: An improved partial similitude method for dynamic characteristic of rotor systems based on Levenberg-Marquardt method. Mech Syst Signal Process 165, $108405(2022)$

41. Zhou, W., Qiu, N., Wang, L., Gao, B., Liu, D.: Dynamic analysis of a planar multi-stage centrifugal pump rotor system based on a novel coupled model. J. Sound Vib. 434, 237-260 (2018)

42. Xu, M., Han, Y., Sun, X., Shao, Y., Gu, F., Ball, A.D.: Vibration characteristics and condition monitoring of internal radial clearance within a ball bearing in a gear-shaft-bearing system. Mech. Syst. Signal Process. 165, 108280 (2022)

43. Wu, J., Zhang, Y., Chen, L., Luo, Z.: A Chebyshev interval method for nonlinear dynamic systems under uncertainty. Appl. Math. Model. 37(6), 4578-4591 (2013) 